\title{
Evolutionary Dynamics Of Service Provisioning
}

\author{
Harry Katzan, Jr., Service Science Institute, USA
}

\begin{abstract}
Business evolves according to market conditions, based on availability and choice. The purpose of this paper is to develop a model that describes how service provisioning evolves through a metaphor of selection, survival of the fittest, replication, and mutation. Clients are free to choose a service provider within a provider category, and the model describes and delineates that behavior. Equations and examples are given.
\end{abstract}

Keywords: Service Provisioning; Evolution; Fitness; Replication; Mutation

\section{INTRODUCTION}

$\mathrm{t}$ is well established that service is a relationship between a provider and a client that captures value. (Spohrer 2007, Katzan 2008, Lusch 2008) It has also been recognized that service is a process, and that all products may in fact be services. (Vargo 2009) This perspective gives us a wide-open playing field for investigating the evolutionary dynamics of service provisioning in particular areas of concern. The purpose of this paper is to develop a model (i.e., a set of equations and relationships) that describes how service evolves through a metaphor of selection, survival of the fittest, replication, and mutation. We will be dealing with categories of service providers, because as Martin Nowak aptly puts it, "Evolutionary dynamics acts on populations. Neither genes, nor cells, nor individuals evolve; only populations evolve." (Nowak 2005) In fact, the model has implications for service provisioning within a service collective. (Katzan 2010b) A client selects a service provider within a particular category for a variety of reasons that pertain to the overall problem domain. As conditions change, the client may select another provider in the same category or switch to a provider in another category. In fact, the client may leave the service system altogether. This paper seeks to apply evolutionary dynamics to a population of service elements. In this instance, the service system responds to a population of clients that serves as the environment in a metaphor of service evolution.

\section{Evolutionary Service Elements}

You don't have service if you don't have clients, also known as customers or consumers, depending upon the context. Clients often have a choice among providers over a particular service domain. Some everyday examples will set the scene. In the medical area, for example, a patient could choose from the categories of medical doctor, osteopathic physician, or chiropractor. This might be regarded as a pure service, as would be the area of education composed of private school, public school, and tutoring. An example of the domain of "services that involve a product" could be a fast-food restaurant offering hamburgers, fish sandwiches, chicken sandwiches, and salads. A bakery providing bread, donuts, muffins, and cake would represent examples of a "product as a service," as would be an automobile company. A familiar means of representing a set of categories is a frame of discernment, as in the following list:

\footnotetext{
Medical $=$ \{medical doctor, osteopathic physician, chiropractor $\}$

Fast food $=$ \{hamburger, fish sandwich, chicken sandwich, salad $\}$

Bakery $=$ bread, donut, muffin, bagel, cake $\}$

Automobile $=\{$ sedan, coupe, convertible, van, suv $\}$

Education $=$ public, private, tutor
} 
The key point is that the client has a free choice within in a service domain. Usually, the choice may change but not the domain. Thus, we have populations of service providers categorized by definitive attributes. We are going to study the evolution of the categories, as a biological scientist might study a species. Within a specific domain, members of a category have common attributes with enough variety to allow for evolutionary service elements, such as natural selection, replication, and mutation.

\section{Elementary Operations}

A client enters and leaves a service system, regarded as a collection of resources, economic entities, and supplementary services capable of engaging in and sustaining one or more service events. (Katzan 2010a) The client selects a service provider from among providers in a relevant service category that possesses sufficient requisite variety to enable that choice. (Ashby 1964) For example, a client may choose a particular physician from a medical-doctor category over a medical domain, a laptop computer over a computer domain, a kind of hamburger over a fast food domain, or a kind of vehicle over an automobile domain. We are concerned about the result of client behavior in such situations.

Within a broad service domain, such as fast food or medical treatment, the client has the choice of behaviors, such as the following:

- $\quad$ Stay with that service category (perhaps comprised of diverse providers)

- $\quad$ Change category

- $\quad$ Leave the service system altogether

A service provider category encapsulates multiple services in the following manner: (1) A single provider performs multiple services; (2) A client can use a single provider for different service events; and (3) A client can move between providers in the category.

There is customarily movement by clients between service categories depending upon social and other metaphysical considerations, for which there is normally a cost and benefit accrued by changing categories. An organization, for example, may change consulting services or cloud service providers. Some categories within a service domain are more attractive for a variety of reasons, collectively identified as "fitness." When clients select a category of service, they are exercising the right to choose, known as democratization. Thus, the process of independent choice, otherwise known as selection, contributes to the fitness of a category with the underlying assumption that clients select the most attractive service provider, based on their temporal needs.

Service dynamics acts on populations of service providers within a particular service domain, so we will be viewing service through a service category lens. Service categories effectively "steal" from other categories by being more fit for selection and replication. The precise reasons that clients move between categories are not relevant to this form of analysis. The important thing is that they do it based on evolutionary considerations. In fact, even the client set is not important for this analysis, since, as mentioned, it serves as the environment for the provider set. We are solely interested in the kinematics of the behavior of populations of service providers.

\section{Biological Metaphor}

In this manner of analysis, there are two populations: a set of clients and a set of providers. A client selects from the available providers. We are not necessarily concerned with individual clients, except for the fact that they select a service category - of course, over a service domain with an associated frame of discernment.

We are going to refer to the collection of service providers in a service domain as a population, comprised of service categories, referred to a species. Species evolve and attract more clients. Species also regress and lose clients. Evolution progresses through selection and replication. Regression can be viewed as the result of a lack of fitness. 
Fitness is a complex phenomenon linked to the ability of a service category's ability to attract clients and evolve in a purposeful manner. An important component of fitness is a service metric, such as market share, sales volume, or revenue. This subject is covered in the next section.

Service categories further evolve through combination and division. New service categories can be formed by combining two or more constituent categories, sometimes known as merging. Service division is a process by which an established service category is divided to form competing categories or supply supplementary services.

As in biological processes, the essence of service dynamics is competition. Categories compete to enhance their values of the service metric and to sustain their position in the chain of categories in a service collective. (Katzan 2010b) As a focal point, one may regard provider behavior as reflecting "market share" and "sustainability."

\section{Democratization And Monetization Of Services}

In the marketplace of services, providers compete for market share, volume, or revenue because there is a limited resource pool (scarcity) or a controlled growth in the availability of required resources. The selection process is a measure of fitness. The clients essentially vote for the provider that is most fit by selecting a specific service category, known as the democratization of services.

Once a service metric is selected, it is important to recognize a dependency on whether the population is fixed or variable. If the birth rate $(\boldsymbol{b})$ and death rate $(\boldsymbol{d})$ of clients is equal, i.e., $\boldsymbol{b}=\boldsymbol{d}$, the methodology, related to fitness, is adjusted from that of a dynamic population, where $\boldsymbol{b}>\boldsymbol{d}$ or $\boldsymbol{b}<\boldsymbol{d}$. For example, if the purchaseability of a set of vehicle buyers is fixed, e.g., one vehicle per family, then a move from sedan to utility vehicle, for example, changes the service metric from the case where the client supplements a sedan with a utility vehicle.

Monetization of services refers to the "survival of the fittest" notion, such that a provider category can adjust its service provisioning in an attempt to change its market position. The form of modeling presented in this paper necessarily applies to both the democratization and monetization of service.

\section{EVOLUTIONARY BEHAVIOR}

Following Nowak (2006) and Olfati-Saber (2007), we seek to develop a formal description of client behavior as elements move between service providers over a frame of discernment. The provider network can be viewed as a complete graph $\mathrm{G}=(\mathrm{N}, \mathrm{E})$, where the nodes $(\mathrm{N})$ represent the case where a set ;of clients enlist the services of a particular set of service providers, and the edges (E) represent movement between the providers resulting from socioeconomic conditions.

\section{Service Demographics}

The methods of analysis are based on a collection $\mathrm{P}$ of $n$ service provider groups over an area of endeavor A. The expectation is that a client will select of member of $\mathrm{P}$ for service. For example, a patient will choose among the distinct categories of medical doctors, osteopathic physicians, or chiropractors for treatment. Each category may incorporate one of more providers. Similarly, a customer of a bakery shop may choose a donut, muffin, or bagel using the "all products are essentially services" (Vargo 2009) conjecture - where in fact there are several choices on the shelf.

Represent the categories of population P over domain A as the vector

$\mathrm{C}=\left[\mathrm{c}_{\mathrm{i}}\right], i=1,2, \ldots, n$

The proportion of the client population that selects a particular provider category is denoted by the vector

$\mathrm{X}=\left[\mathrm{x}_{\mathrm{i}}\right], i=1,2, \ldots, n$ 
where $x_{i}$ represents the proportion of the client population associated with provider category $c_{i}$, where $\sum_{i} x_{I}=1$. The analyst may interpret the metric $\mathrm{X}$ in any manner pertaining to the analysis, such as the number of clients, total value of client interactions, or the volume of client service.

The attitudes and beliefs resulting from prior service event or interactions with other clients affect the $x_{i}$. At this stage of the research, we are not going to consider client movement within a category.

Applying the biology metaphor, the collection of provider categories $\mathrm{C}$ are a species in the population $\mathrm{P}$, and a category replicates in response to client requirements - or more accurately, client preferences.

An example of this form of replication would be innovation and competition based on increasing market opportunities. The notion of fitness applies here as the most-fit providers attract the most clients.

\section{Service Kinematics} state vector:

The status of a service system, under consideration at a given point in time, is therefore represented by the

$\mathrm{X}=\left[\mathrm{x}_{1}, \mathrm{x}_{2}, \ldots, \mathrm{x}_{\mathrm{n}}\right]$

where each $x_{i}$ reflects the proportion of clients that choose provider category $c_{i}$. Consider a reward matrix $A=\left[a_{i j}\right]$ that represents the reward that a client would obtain by switching from category $c_{j}$ to category $c_{i}$. Thus, a service category replicates in response to client preferences. One could conceptualize a business switching for computer consulting services from one provider to another, but only if there is some measurable utility for doing so. When a provider $c_{i}$ replicates, the value of its state value $x_{i}$ increases based on a fitness measure determined by the values in the reward matrix.

\section{Mutation}

Mutation is an important element in replication based on fitness. The probability at clients in group $c_{i}$ mutates to group $c_{j}$ is given by matrix:

$\mathrm{Q}=\left[\mathrm{q}_{\mathrm{ij}}\right]$, where $0 \leq \mathrm{q}_{\mathrm{ij}} \leq 1$

Q is square row stochastic matrix of order $n \times n$ and $\Sigma_{j}^{n} q_{i j}=1$. Thus, $q_{i j}$ can be regarded as the rate of transference from $\mathrm{x}_{\mathrm{i}}$ to $\mathrm{x}_{\mathrm{j}}$ based on exogenous conditions.

\section{SERVICE DYNAMICS}

Several equations, adapted from mathematical biology, Nowak (op cit.) and Olfati-Saber (op cit.), are used to describe client behavior. In order to properly frame the problem of service dynamics, several considerations are useful for explaining the terminology adapted from evolutionary biology. As with most instances of socioeconomic behavior, some service providers dominate because there is a positive utility for their selection. The term commonly used to reflect a position is "fitness." The service providers that are most fit receive the attention, and not-sofortunate providers adapt to better compete and increase their sustainability index. We are going to base the fitness metric on the reward matrix A, covered earlier. Mutation is also introduced, because even though clients are assumed to be rational, other considerations from the environment often apply.

\section{Fitness}

Fitness is a measure of an element $c_{i}$ from category $\mathrm{C}$ based on the reward matrix $\mathrm{A}$. The fitness vector is $\mathrm{F}$ $=\left[\mathrm{f}_{\mathrm{i}}\right]$, where $\mathrm{i}=1,2, \ldots, n$, and is computed as:

$f_{i}=\Sigma_{j} a_{i j} x_{j}$ 
and its average $\Phi$ for category $\mathrm{C}$ is computed as:

$\Phi=\Sigma_{\mathrm{i}} \mathrm{x}_{\mathrm{i}} \mathrm{f}_{\mathrm{i}}$

Clearly, the notion reflects the reward of switching from $c_{j}$ to $c_{i}$.

The types $c_{i}$ are competing for resources from the environment of clients, where for example, one service category evolves faster, based on the reward matrix for clients and out-competes other service providers.

The basic measure of fitness is the degree to which a category attracts clients, represented by the column $i$ of the matrix, resulting in equation [1].

\section{Service Evolution}

When $f_{i}>\Phi$, the state value $x_{i}$ for $c_{i}$ increases and when $f_{i}<\Phi$ the state value $x_{i}$ for $c_{i}$ decreases. The associated evolution value for $\mathrm{x}_{\mathrm{i}}$ is computed from the following difference equation:

new $\mathrm{x}_{\mathrm{i}}=\mathrm{x}_{\mathrm{i}}\left(1+\left(\mathrm{f}_{\mathrm{i}}-\Phi\right)\right)$

where $f_{i}$ is the fitness of $c_{i}$ and $x_{i}$ is the corresponding state value. The state value $x_{i}$ is the proportion of clients that choose provider $c_{i}$. (Novak 2006, Olfati-Saber 2007) An example of service evolution is given in a succeeding section.

\section{Example of Service Evolution}

The example describes an a service domain comprised of computer vendors, given by the frame of discernment $\mathrm{C}$ :

$\mathrm{C}=\{$ Apple, Sony, Dell $\}$

and the state vector $\mathrm{X}$, as:

$\mathrm{X}=(0.5,0.2,0.3)$

such that $\mathrm{c}_{1}=$ Apple corresponds to a state value of $\mathrm{x}_{1}=0.5$. A hypothetical reward matrix is given as:

$A=\left(\begin{array}{ccc}1 & 1.5 & 2 \\ 0.5 & 1 & 0.25 \\ 0.25 & 0.5 & 1\end{array}\right)$

Each row represents the rewards of migrating to a category (i.e., the row) from the other categories (i.e., the column). Thus, a client would obtain a reward of 1.5 from Sony to Apple and of 2 from Dell to Apple, and so forth. Row one represents Apple, row two represents Sony, and row represents Dell. The fitness vector $F=\left[f_{i}\right]$ is determined as

$\mathrm{F}=(1.4,0.35,0.525)$

from equation [1], where $\Phi$ is computed as 0.82 from equation [2]. Accordingly, the new state vector, referred to as new $X$, is computed from equation [3], as

new $X=(0.79,0.106,0.12)$

reflecting an evolution to the succeeding state. 


\section{Mutation Matrix}

A form of mutation, representing as conversion between states, is modeled as the rpw stochastic matrix $Q=$ $\left[\mathrm{q}_{\mathrm{ij}}\right]$ such that $\Sigma_{\mathrm{j}} \mathrm{q}_{\mathrm{ij}}=1$. The mutation matrix is computed from a weight matrix $\mathrm{W}=\left[\mathrm{w}_{\mathrm{ij}}\right]$, where $\mathrm{w}_{\mathrm{ij}}=\mathrm{a}_{\mathrm{ij}} /\left(\Sigma_{\mathrm{j}} \mathrm{a}_{\mathrm{ij}}\right)$. A mutation parameter $\mu$ is defined, so that $\mathrm{q}_{\mathrm{ij}}=\mu \mathrm{w}_{\mathrm{ij}}$. (Olfai-Saber $\underline{\mathrm{cp} \text { cit.) }}$

Introducing mutation into the modeling, we can develop a more sophisticated replication model as

new $\mathrm{x}_{\mathrm{i}}=\mathrm{x}_{\mathrm{i}}\left(1+\left(\left(\Sigma_{\mathrm{j}} \mathrm{x}_{\mathrm{j}} \mathrm{f}_{\mathrm{j}} \mathrm{q}_{\mathrm{j}}\right)-\Phi \mathrm{x}_{\mathrm{i}}\right)\right)$

Mutation is beyond the scope of this paper and presentation, but the notion of mutation is an intriguing topic for evolutionary modeling.

\section{SUMMARY}

The subject of evolutionary modeling based on the work of Nowack and Olfati-Saber has been introduced, and its application to the evolutionary modeling of service provisioning has been presented. Equations for fitness and replication have been developed that differ from biological processes, but retain the requisite elements for a viable methodology. A relevant numerical example has been included.

\section{AUTHOR INFORMATION}

Professor Harry Katzan, Jr. is the author of books and papers on computer science, decision science, and service science. He teaches in the MBA program at Webster University and is the director of the Service Science Institute. E-mail: katzanh@hargray.com.

\section{REFERENCES}

1. Ashby, W. 1964. An Introduction Cybernetics. London: Chapman and Hall.

2. Katzan, H. 2008. Service Science: Concepts, Technology, Management. New York: iUniverse, Inc.

3. Katzan, H. 2010a. Service Analysis and Design. International Applied Business Research Conference Proceedings, Orlando, FL: IABR Conference, January 4-6, 2010.

4. Katzan, H. 2010b. Service Collectivism, Collaborations, and Duality. International Applied Business Research Conference Proceedings, Orlando, FL: IABR Conference, January 4-6, 2010.

5. Lusch, R., Vargo, S., and G. Wessels. 2008. Toward a conceptual foundation for service science: Contributions from service-dominant logic. IBM Systems Journal, 47(1): 5-14.

6. Nowak, M. 2006. Evolutionary Dynamics: Exploring the Equations of Life. Cambridge, MA: The Belknap Press of Harvard University Press.

7. Olfati-Saber, R. 2007. Evolutionary Dynamics of Behavior in Social Networks. Proceedings of the $46^{\text {th }}$ IEEE Conference on Decision and Control (December 2007).

8. Spohrer, J., Vargo, S., Caswell, N. and P. Maglio. 2007. The Service System is the Basic Abstraction of Service Science. IBM Almaden Research Center, http://www.almaden.ibm.com/asr.

9. Vargo, S. and M. Akaka. 2009. Service-Dominant Logic as a Foundation for Service Science: Clarifications. Service Science, 1(1): 32-41. 